\title{
SUCESSÃO DE ESPÉCIES DE PEIXES EM RECIFES ARTIFICIAIS NUMA ILHA COSTEIRA DO LITORAL SUL BRASILEIRO
}

\author{
JARDEWESKI, C.L.F. ${ }^{1} \&$ ALMEIDA, T.C.M. ${ }^{2}$ \\ Laboratório de Ecologia de Comunidades - Universidade do Vale do Itajaí \\ Centro de Ciências Tecnológicas da Terra e do Mar \\ Av Uruguai, 452. Centro, Itajaí/SC. CEP 88330-002 - Brasil \\ 1'cleitonlfj@yahoo.com.br, ${ }^{2}$ tito@univali.br.
}

\begin{abstract}
Jardeweski, C.L.F. \& Almeida, T.C.M. 2005. Sucession of Fish Species in Artificial Reefs at a Coastal Island in Souh Brasillian Litoral. Braz. J. Aquat. Sci. Technol. 9(2):57-63. ISSN 1808-7035. The utilization of artificial structures placed in marine bottom for fish attraction date of long time. In the last 10 years artificial reefs has been used in brazillian coast under several reasons, like: productivity enhancement, sub-aquatic tourism, fish trawling reduction, etc. However little information was known about the impacts of artificial reef, such positives than negatives, in the first semester of 2004 a law project, in Brazil, was approved liberating the practice by many ecological reasons. The present study was developed in Porto Belo Island, in the shore of Santa Catarina, Brazil. Six unities of ReefBallTM, in two blocks of three unities, were placed near the island. One block was placed near the island rocky shore (RD) and the other block 60 meters far from the rocky shore (RF). Fish visual censuses were done by SCUBA, monthly, since October of 2002 to September of 2003 in both sites. The pattern of species succession in each block was characterized by cumulated curves of species in the time long, and the curves calibrated by the minimum squares and the angular coefficient founded for each one of the sites compared by the Student Test. A Total of 23 species was identified for RD and 8 to RF. The increasing number of species, measured by the angular coefficient of each equation, was significant different $\left(t_{\text {callb1- }}\right.$ $\left.{ }_{b 2}=4,474 ; p<<0,01\right)$ and superior in the block near the rocky shore $\left(b_{R D}=1,503 ; b_{R F}=0,682\right)$. Different raising pattern in the species number could be verified when compare the both succession curves, RF showed two distinct pulses. In RD the raising number of species also was verified however this raise was more gradual and continual. The results indicated that the proximity to other natural subtract act directly on the colonization process by fishes in artificial structures.
\end{abstract}

Keywords: Sucession, Artificial Reefs, Ichthyofauna, Brazil.

\section{INTRODUÇÃO}

Recifes artificiais são cada vez mais utilizados das mais variadas formas e finalidades. A sua utilização vai desde o ambiente límnico, tais como reservatórios e barragens (Freitas \& Petrere, 2001; Seaman, 1995), ao marinho, tanto em regiões costeiras (Fenessy et al, 1998) como oceânicas (Holbrook et al, 2000). Dentre os diversos propósitos destacam-se: o incremento de pesca artesanal (Conceição, 1998; Brandini, 1999; Phongsuwan et al, 1994), controle de erosão praial (Gradner et al, 1996), proteção de áreas de relevante interesse ecológico (Rilov \& Benayahu, 1998), novas alternativas para mergulho (Brock, 1994), exploração petrolífera (Hostim-Silva et al, 2002; Holbrook et al, op. cit.) e maricultura (Figna, 2002; Fabi \& Fiorentini, 1996).

As pesquisas em recifes artificiais geralmente tiveram uma conotação prática, ou seja, voltadas à construção, aos materiais utilizados, ao desenho das estruturas, sendo que as de cunho ecológico foram colocadas em segundo plano (Jardeweski \& Almeida, no prelo; Svane \& Petersen, 2001). Com isto há uma grande deficiência nas pesquisas realizadas, questões tróficas e de produção são temas pouco abordados, assim como estudos quantitativos, experimentais e energéticos são praticamente inexistentes na literatura especializada (Svane \& Petersen, 2001; Godoy \& Coutinho, 2002), 0 que dificulta muito o entendimento dos períodos iniciais de sucessão em recifes artificiais.

A maioria dos estudos concentra-se sobre a comunidade de peixes, principalmente através da metodologia de censo visual (Jardeweski \& Almeida, op cit; Rilov \& Benayahu, 1998; Godoy \& Coutinho, 2002). Somente esta metodologia pode demonstrar diversos aspectos relacionados a colonização, sucessão, utilização do espaço e respostas comportamentais da ictiofauna. Todos fundamentais ao entendimento e ao estudo da ecologia de peixes em recifes artificiais.

Nakamura (1985, apud Athiê, 1999) estudou e adaptou a classificação do ARRG (Artificial Reef Research Group) para peixes associados a recifes artificiais, quanto à resposta comportamental e ao aproveitamento do recife por parte dos peixes, categorizando estes em três tipos distintos:Tipo I - peixes que preferem o contato físico com o recife, ocupando buracos, fendas e aberturas estreitas, sendo predominantemente bentônicos, chamados de colonizadores;Tipo II - 
peixes que nadam próximo ou entre os recifes artificiais, próximos ao fundo, estando ligados a estrutura através da visão e do som, chamados de exploradores;Tipo III - peixes que tendem a nadar por cima ou ao largo do recife enquanto permanecem nas porções média e superior da coluna d'água, chamados de ocasionais.

O presente estudo acompanhou a sucessão de peixes na colonização de recifes artificiais junto e distantes de um costão rochoso numa ilha costeira em Santa Catarina, e teve como objetivo determinar, descrever e comparar o padrão de diversidade ao longo do tempo.

\section{MATERIAIS E MÉTODOS}

\section{Área de Estudo}

O trabalho foi desenvolvido na região de Porto Belo, na Ilha João da Cunha/SC, localizada a 2709' 28"S e 48 33' 11"W (Figura 1a). Durante o período de outubro de 2002 a setembro de 2003. A região se caracteriza como uma baia estável e bem abrigada. Entre a porção sul e sudeste da ilha, e os costões da região conhecida como "Araçá", forma-se um canal influenciado principalmente pela maré, que atua diretamente em toda a hidrologia da região.

$\mathrm{Na}$ baia existem algumas áreas de cultivo de moluscos as quais favorecem a redução no hidrodinamismo, o que eleva as taxas de sedimentação e reduzem a visibilidade da água. O sedimento na região, de modo geral, se caracteriza por uma mistura de areia e lama, o que também contribui para a redução na visibilidade.

Dois grupos, de três estruturas cada, foram estabelecidos e monitorados em dois locais distintos: um próximo ao costão da ilha (RD) e outro colocado a uma distancia de 50 metros do mesmo local (RF) (Figura 1b) .

\section{Censo Visual}

O monitoramento da comunidade de peixes foi efetuado por meio de censos visuais realizados ao longo de transectos lineares (Sanderson \& Solonsky, 1986; Greene \& Alevizon, 1989; Watson \& Quinn II, 1997), de dimensões $20 \times 2 m$ num período de 10 minutos, dispostos aleatoriamente em "zig-zag" nos três locais mencionados. Esta forma de realização dos censos foi adaptada devido às limitações logísticas em campo, tais como visibilidade e posicionamento entre os recifes no fundo.

Os censos foram realizados mensalmente, através de mergulho autônomo, em cada censo, todos os peixes eram contados ao longo dos transectos, e as identificações eram feitas durante os censos até o menor táxon possível, seguindo a classificação de Figueiredo \& Menezes (1980). Os peixes eram classificados quanto às espécies e anotados em pranchetas subaquáticas pelos censores. As áreas eram monitoradas no mesmo dia e período, para não haver grande variação das condições ambientais.

\section{Análise dos Dados}

O padrão de sucessão de espécies em cada um dos blocos foi caracterizado através de curvas de espécies acumuladas ao longo dos meses, sendo as retas de ajuste calculadas por meio dos mínimos quadrados e os coeficientes angulares, de cada uma das retas comparados por meio de um teste $t$ de Student (Zar, 1984). As espécies foram classificadas segundo Nakamura (1985, Athiê, 1999) e representadas através de curvas de espécies acumuladas ao longo dos meses.

\section{RESULTADOS}

Um total de 23 táxons foi identificado para o bloco de recifes dispostos junto ao costão (RD) e 8 para o bloco distante do costão (RF) (Tabela 1). Stegastes fuscus foi a espécie mais freqüente em RD, ocorrendo em todos os meses, seguido por Myctiroperca acutirostrise Diplodus argenteus que ocorreram em 10 dos 12 meses amostrados. Em RF Haemulon sp e Mictiroperca acutirostrix, foram as espécies mais abundantes, ocorrendo em 6 dos 12 meses estudados (Tabela 1).

Através da classificação de Nakamura (apud Athie, 1999), também foram feitas as curvas de espécies acumuladas para cada tipo e local (Figura 2). Em ambos os locais, houve o aumento do número de espécies ao longo do tempo, tanto para o tipo I como para o tipo II, somente as espécies ocasionais (tipo III) não tiveram registro em $\mathrm{RF}$, somente uma ocorrência em $\mathrm{RD}$ no mês de abril. Em ambos os locais as espécies exploradoras (Tipo II) foram as primeiras a serem registradas em RF, sendo o número de indivíduos deste tipo comportamental igual ou maior que o do tipo I, predominando durante todo o experimento. Em RD as espécies colonizadoras, a partir do terceiro mês, permaneceram em maior número até o final do monitoramento.

As curvas de espécies acumuladas construídas para RD e RF apresentaram padrões diferentes na sucessão de espécies (Figura 2). Em RF, o aparecimento das espécies ocorreu na forma de pulsos (Figura 2 A), enquanto que em RD ocorreu de forma mais gradu- 


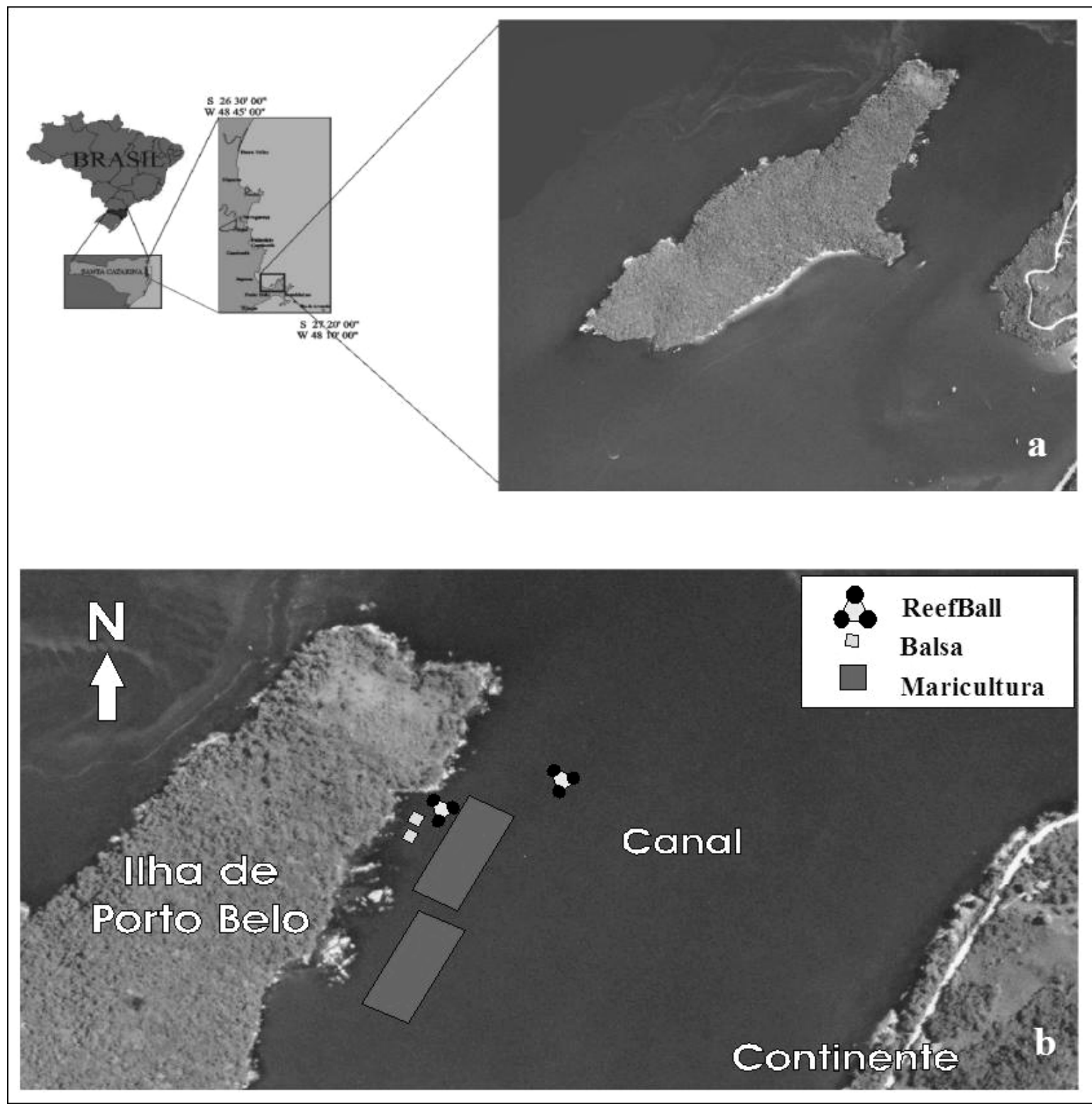

Figura 1 - a) Ilha João da Cunha, b) Localização dos Recifes Artificiais.

al (Figura 2 B). Os parâmetros estatísticos das retas de ajustes de RD e RF (a, b e $R^{2}$ ) foram significativos (Tabela 2) e os coeficientes angulares quando comparados apresentaram diferença significativa $\left(\mathrm{t}_{\text {calc }}=4,474\right.$; $\mathrm{p}<0,01)$.

\section{DISCUSSÃO}

Os resultados obtidos demonstraram o padrão que ocorre na colonização por peixes ao longo do tempo em recifes artificiais, do tipo "ReefBall ${ }^{\mathrm{TM}}$ ". Foi observado o aumento no número de espécies e na abundância de indivíduos, tanto nos recifes junto ao costão quan- to nos distantes. Resultados semelhantes foram obtidos por Athiê (1999).

No processo de sucessão nos recifes, foi verificado que $\mathrm{RD}$ apresentou uma colonização mais rápida e expressiva que RF. Embora Athiê (1999) não tenha obtido assíntota nas curvas de espécies acumuladas construídas para seis estruturas artificiais monitoradas 210 dias, em ambos os locais aqui estudados parecem estabilizar próximo ao final de um ano. Este autor atribui ao tempo o fator mais importante na estruturação da comunidade de peixes, no entanto a proximidade com um local fonte, como um costão rochoso (RD), pode influenciar no processo de colonização. 
Tabela 1 - Freqüência de espécies encontrados nos recifes artificiais, e a classificação de Nakamura (Tipo 1 - colonizadora, Tipo II exploradora, Tipo III - ocasional).

\begin{tabular}{llccc}
\hline Taxa & Nome popular & Freq. RD & Freq. RF & Tipo \\
\hline Blenniidae & Maria da Toca & $8 \%$ & & 1 \\
Acanthostracion quadricornis & Peixe Cofre & $8 \%$ & & 3 \\
Anisotremus virginicus & Salema & $8 \%$ & $25 \%$ & 1 \\
Bhathygobius soporator & Maria da Toca & $8 \%$ & $42 \%$ & 2 \\
Caranx sp & Xerelete & $8 \%$ & & 2 \\
Chaetodon striatus & Peixe Borboleta & $33 \%$ & & 1 \\
Diodon sp & Baiacu de Espinho & $8 \%$ & & 2 \\
Diplectrum sp & Aipim & $50 \%$ & $25 \%$ & 2 \\
Diplodus argenteus & Marimbau & $42 \%$ & & 2 \\
Epinephelus marginatus & Garoupa & $8 \%$ & & 1 \\
Epinephelus morio & Garoupa São Tomé & $8 \%$ & & 2 \\
Epinephelus niveatus & Cherne & $100 \%$ & & 1 \\
Pareques acuminatus & Peixe Gato & $17 \%$ & & 1 \\
Eucinostomus sp & Escrivão & $8 \%$ & $17 \%$ & 1 \\
Gymnothorax ocellatus & Moreia pintada & $8 \%$ & & 2 \\
Haemulon aurolineatum & Corcoroca & $8 \%$ & $50 \%$ & 1 \\
Myctiroperca acutirostris & Badejo Mira & $33 \%$ & $58 \%$ & 1 \\
Ogcocephalus vespertilio & Peixe Morcego & $17 \%$ & & 1 \\
Scorpaena sp & Mangangá & $8 \%$ & $17 \%$ & 1 \\
Sparisoma sp & Peixe Papagaio & $83 \%$ & & 1 \\
Sphoeroides testudineus & Baiacu & $33 \%$ & & 2 \\
Stegastes fuscus & Donzelinha & $83 \%$ & & 2 \\
Stephanolepis hispidus & Peixe Porco & $50 \%$ & $17 \%$ & 2 \\
\hline
\end{tabular}

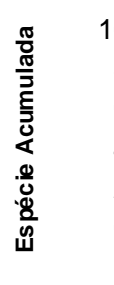

A

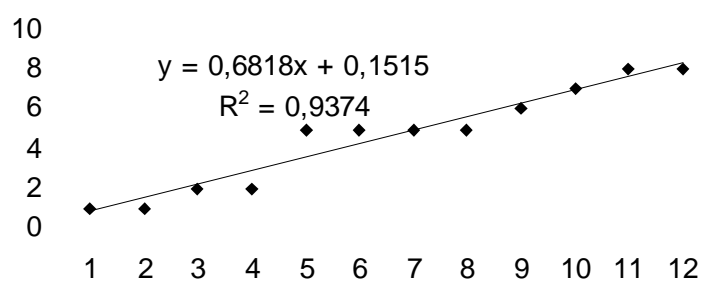

Meses

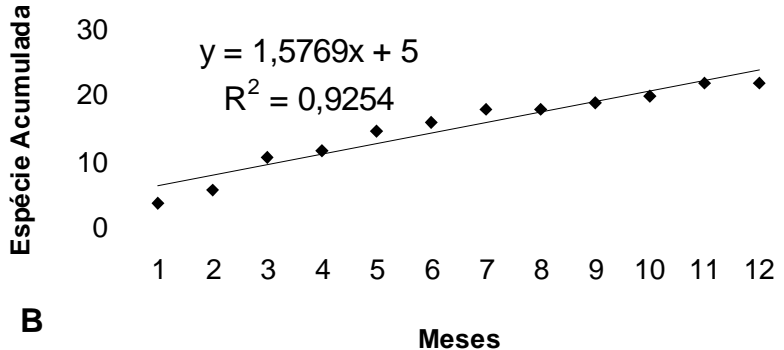

Meses

Figura 2 - Curvas de Taxas acumuladas nos recifes de fora (A) e nos junto ao costão (B).

Tabela 2 - Valores dos coeficientes: linear (a) e angular (b), e respectivos coeficientes de determinação das curvas de RF e RD $\left({ }^{* * *}\right.$ representam $\left.p<0,00001\right)$.

\begin{tabular}{lccc}
\hline \hline & $\mathrm{a}$ & $\mathrm{b}$ & $\mathrm{R}^{2}$ \\
\hline $\mathrm{RF}$ & 0,1515 & $0,6818^{\star \star *}$ & $0,937^{\star \star \star}$ \\
$\mathrm{RD}$ & $5^{* * *}$ & $1,5769^{* * *}$ & $0,925^{\star * *}$ \\
\hline \hline
\end{tabular}

A colonização na forma de pulsos, verificada em RF, pode ser atribuída a fatores sazonais hidrológicos relativos ao local e das espécies que iniciaram a colonização. Barreiros et al (2004), encontrou padrões de variações sazonais e na diversidade da ictiofauna em poças de maré na costa de Santa Catarina, relacionados com fatores hidrológicos sazonais.

Este último fator ficou demonstrado nos primeiros momentos de colonização, pois as primeiras espécies que apareceram, em RF, foram às classificadas 

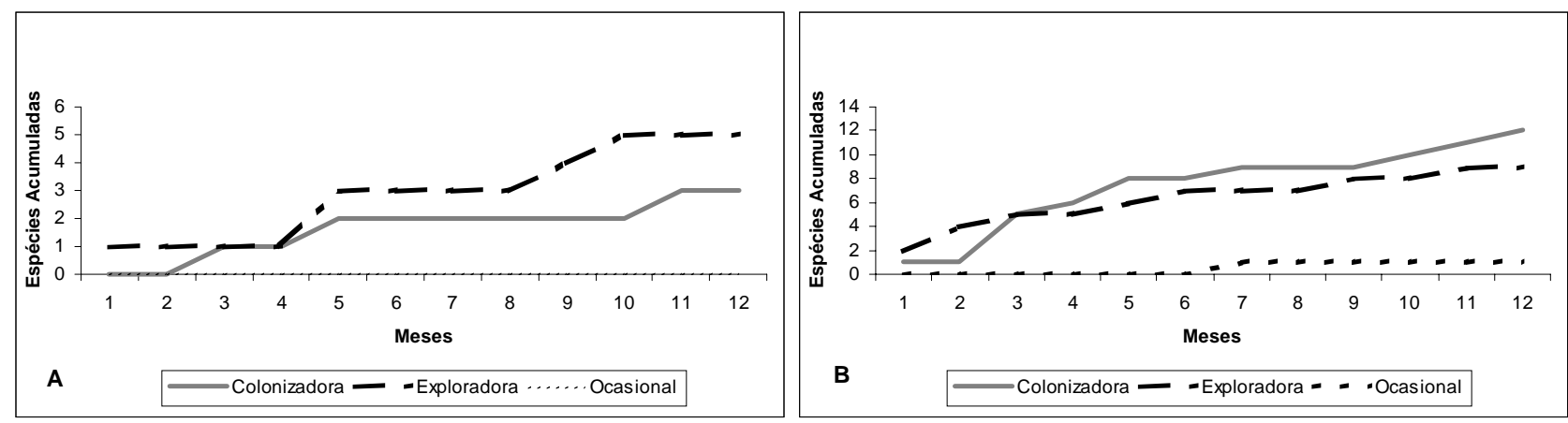

Figura 3 - Curvas de Espécies acumuladas, segundo classificação de Nakamura, nos recifes de fora (A) e nos junto ao costão (B).

segundo Nakamura em 1985 (apud, Athie) exploradoras (tipo II), e somente a partir do terceiro mês as espécies classificadas como colonizadoras (tipo I) começaram a surgir e formar uma associação de espécies sedentárias. Em RD desde os primeiros meses os dois principais tipos (I e II) sempre ocorreram e foram aumentando a diversidade através do tempo, denotando o crescimento gradual e continuo da curva de espécie acumulada. A classificação de Nakamura (1985), aplicada sobre a ictiofauna, corrobora estes fatos, uma vez que as espécies do tipo I (colonizadoras), em RD, após dezembro apresentaram pouca ou nenhuma variação em sua densidade representando a ocupação rápida dos nichos na comunidade. Já em RF verificou-se que estas espécies somente a partir de fevereiro foram encontradas, apresentando neste local maior variação na densidade do que em RD.

Quando comparamos as curvas de espécie acumulada com as da classificação de Nakamura, podemos notar que o mesmo padrão é mantido, tanto em RD como em RF. Portanto na colonização não houve diferenças nos padrões de colonização entre os tipos comportamentais de peixes, demonstrando que a colonização inicial independe da resposta comportamental e do aproveitamento do recife por parte da ictiofauna.

Comparando os gráficos da Figura 2 com os da Figura 3 podemos constatar que os padrões de colonização são mantidos independentemente do tipo comportamental ou de aproveitamento dos peixes nos recifes artificiais. Pois os pulsos de colonização encontrados em RF, e o aumento gradual continuo ilustrado em RD (Figura 2) são mantidos; não havendo diferença na sucessão mesmo quando separamos os peixes pela classificação de Nakamura (Figura 3). Através deste resultado podemos aferir que os períodos iniciais de sucessão independem da resposta comportamental ou do aproveitamento do recife por parte da ictiofauna.

A colonização verificada demonstra que a sazonalidade hidrológica do local foi uma das principais características que influenciaram a sucessão em RF, demonstrando que a escolha dos locais onde são colocados recifes artificiais se mostrou como um importante fator na estruturação de uma nova comunidade de peixes.

Seguindo estes fatos podemos afirmar que: 0 local onde são colocados os recifes artificiais se mostrou como um importante fator na estruturação de uma nova comunidade de peixes. Esta característica espacial delimitou a atração de novos indivíduos para ambos os locais em que foram colocadas as estruturas.

\section{REFERÊNCIAS}

Athie, A.A.R. 1999. Colonização e Sucessão Ecológica de Peixes em Recifes Artificiais no Canal de São Sebastião, Litoral Norte do Estado de São Paulo - Brasil. Dissertação de Mestrado, IOUSP.

Athie, A.A.R. 1999. Recifes Artificiais: Ciência e Tecnologia Emergente no Brasil. VII Congresso Latino Americano de Ciências do Mar, anais, 54-56.

Barreiros, J.P.; Bertoncini, A.; Machado, L.; HostimSilva, M. \& Santos, R.S. 2004. Diversity and seasonal changes in the ichthyofauna of rocky tidal pools from Praia Vermelha and São Roque, Santa Catarina. Brazilian Archives of Biology and Technology, vol 47. n.2: 291-299.

Bohnsack, J. A.; Ecklund, A.M. \& Szmant, A.M. 1997. Artificial Reef Research: Is There More Than The Attraction-Production Issue? Fisheries, Special Issue on Artificial Management, vol 22, $\mathrm{n}^{\mathrm{O}} 4$.

Brandini, F.A. 1998. Utilização de Recifes Artificiais para o Desenvolvimento de Comunidades Marinhas e seus Benefícios Sócio Econômicos. IV Simpósio de Ecossistemas Brasileiros: Mesa Redonda, Águas de Lindóia, São Paulo.

Brock, R.E. 1994. Beyond Fisheries Enhancement: Artificial Reefs and Ecotourism. Bulletin of Marine Science 55(2-3), 1181-1188.

Carr, M.H. 1994. Effects of Macroalgal Dynamics on the Recruitment of a Temperate Reef Fish. Ecology, $75,1320-1333$. 
Carr, M.H. \& Hixon, M.A. 1997. Artificial Reefs: The Importance of Comparisons with Natural Reefs. Fisheries: Special Issue on Artificial Reef Management, vol $22 \mathrm{n}$ 0. 4, 28-33.

Conceição, R.N. de L. \& Monteiro, C. 2002. Recifes Artificiais Marinhos - Incrementando a Pesca nas Comunidades Costeiras do Ceará - UFCE. Disponível em: < http://www.biotecnologia.com.br/bio/ 6 b.htm >, versão 13/12/02.

Ebeling, A.W. \& Laur, D.R. 1985. The Influence of Plant Cover on Surfperch Abundance at an Offshore Temperate Reef. Environmental Biology of Fishes, 12, 169-179.

Ferreira, C.E.L.; Gonçalves, J.E.A. \& Coutinho, R. 1993. Metodologias em Estudos de Comunidades de Peixes em Costões Rochosos: Questões Básicas e Fundamentais. III Simpósio de Ecossistemas da Costa Brasileira, ACIESP.

Figna, V.D.N. 2002. Aspectos Bioecológicos dos Peixes Associados a Maricultura na Ilha de Porto Belo, Porto Belo, Santa Catarina - Brasil. Trabalho de Graduação - UNIVALI.

Freitas, C.E.C. \& Petrete Jr, M 2001. Influence of Artificial Reefs on Fish Assemblages of the Barra Bonita Reservoir (São Paulo, Brazil). Lakes \& Reservoirs: Research and Management 6, 273-278.

Froese, R. \& Pauly, D. 2003. FishBase. World Wide Web electronic publication. www.fishbase.org, version 24 September 2003.

Godoy, E.A.S.; Almeida, T.C. M. \& Zalmón, I.R. 1998. Fish Assemblages and Environmental Variables on an Artificial Reef - Rio de Janeiro, Brazil. Journal of Marine Science, 59.

Godoy, E.A. de S. 2000. Fauna Associada a Bancos Artificiais de Sargassum Furcatum na llha de Cabo Frio - RJ, Brasil. Dissertação de Mestrado - UENF.

Godoy, E.A. de S. \& Coutinho, R. 2002. Can Artificial Beds of Plastic Mimics Compensate for Seasonal Absence of Natural Beds of Sargassum furcatum? Journal of Marine Science 59, 111-115.

Grossman, G.D.; Jones, G.P. \& Seaman, W.J. 1997. Do Artificial Reefs Increase Regional Fish Production? A review of Existing Data. Fisheries, Special Issue on Artificial Management, vol 22, $\mathrm{n}^{\mathrm{O}}$. 4.

Holbrook, S.J. \& Schmidt, R.J. 1994. Spatial And Temporal Patterns In Assemblages Of Temperate Reef Fish. American Zoologist 34, 463-508.

Hostim-Silva, M. ; Fontes, J.; Afonso, P; Serpa, N.; Sazima, C.; Barreiros, J. P. \& Sazima, I. 2002. Plataformas de Petróleo - Pontos de Encontro de Peixes em Alto-Mar. Ciência Hoje, vol 31, n ${ }^{0} 183$.

Jardeweski, C.L.F. \& Almeida, T.C.M. in press. Fish Assemblage on Artificial Reefs on South Brazilian
Coast. Journal of Coastal Research, SI 39 (Proceedings of the $8^{\text {th }}$ International Coastal Symposium), Itajaí, SC - Brazil.

Figueredo J.L. \& Menezes, N. 1980. Manuais de peixes marinhos do sudeste do Brasil. III. Teleostei. Museu de Zoologia. Universidade de São Paulo. 90p.

Oren, O.H. 1968. Artificial Reefs - A Short Review And Appeal, Fao (Food And Agriculture Organization Of The United Nations) Roma.

Phongsuwan, N.; Chansang, H. \& Satapoomin, U. 1994. Colonization of Fouling Communities and Associated Fauna at the Artificial Reefs. The Effect of Artificial Reef Installation on the Biosocioeconomics of Small-scale Fisheries in Ranong Province, Thailand. 17-27.

Pitcher, T.J. \& Seaman, W. 2000. Petrarch's Principle: How Protected Human-Made Reefs Can Help The Reconstruction of Fisheries and Marine Ecosystems. Fish and Fisheries 1, 73-81.

Reefball, Programa Recifes Artificiais Marinhos, Uma Proposta De Conservação Da Pesca Artesanal Na Costa Do Estado Do Paraná, Instituto Ecoplan e Centro De Estudos Do Mar - CEM / UFPR. 1998. Disponível Em: <http://www.reefball.com/ecoplan/ index.html> , versão 14/10/1998.

Rilov, G. \& Benayahu, Y. 2000. Fish Assembalge On Natural Versus Artificial Reefs: The Rehabilitation Perspective. Marine Biology.

Santos, M.N. \& Monteiro, C.C. 1997. The Olhão Artificial Reef System (South Portugal): Fish Assemblages and Fishing Yield. Fisheries Research 30, 33-41.

Saul, A.C. \& Cunningham, P.T.M. 1997. Use Of Space By Fish In Artificial Reefs In Brazil: An Experimental Approach. VII Colacmar, P. 418-419, Santos, São Paulo.

Seaman, W. 1995. Artificial Habitats for Fish. Enciclopedia of Environmental Biology, 93-104.

Spanier, E.; Tom, M.; Pisanty, S. \& Almog-Shtayer, G. 1990. Artificial Reefs In The Low Productive Marine Environments Of The Southeastern Mediterranean. Marine Ecology, 11(1): 61-75.

Summerhayes, C.P. \& Thorpe, S.A. 1996. Oceanography an Illustrated Guide, SouthamptonUk, Manson Publishing - P.259-272.

Svane, I. \& Petersen, J.K. 2001. On The Problems of Epibioses, Fouling and Artificial Reefs, a Review. Marine Ecology 22, 169-188.

Watson, R.A. \& Quinn II, T.J. 1997. Performance of Transect and Point Count Underwater Visual Methods. Ecological Modelling 104, 103-112.

Wegner, E. 2002. Proposta Metodológica Para Implantação de Trilhas Subaquáticas na llha de Porto Belo, Porto Belo SC. Dissertação de Mestrado - UNIVALI. 
Braz. J. Aquat. Sci. Technol., 2005, 9(2):57-63.

Willis, T.J. 2001. Visual Census Method Underestimate Density and Diversity of Cryptic Reef Fishes. Journal of Fish Biology 59, 1408-1411.
Zar, J. H. 1984. Bioestatistical analysis. Prentice Hall Ed. $718 \mathrm{pp}$.

Submetido: Outubro/2004 Revisado: Fevereiro/2005 Aceito: Agosto/2005 